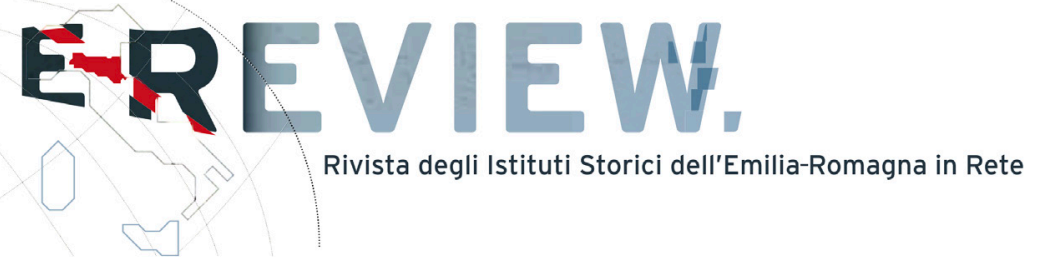

$\frac{\text { 8-9, 2021-2022 }}{\text { \#dossier }}$ 
E-REVIEW

Rivista degli Istituti Storici dell'Emilia-Romagna in Rete

Andrea Ceci

Itinerario nella monumentalità risorgimentale

emiliano-romagnola tra spirito nazionale e identità locali

(C) Andrea Ceci

Creative Commons BY-NC-ND 4.0

International License 2004-2021

Volume: 2021-2022

Issue: 8-9

Section: \#dossier - sul piedistallo della storia

Pages: 1-28

DOI: $10.52056 / 9788833138756 / 01$

ISSN: 2282-4979

Publisher: Viella

Double blind peer review: Yes

Document type: Article

Research Areas: History

Published: December 2021

Corresponding Address: Andrea Ceci, Università di Pisa, Dipartimento di Civiltà e Forme del Sapere, via Pasquale Paoli 15, 56126 Pisa, Italy 


\title{
Itinerario nella monumentalità risorgimentale emiliano-romagnola tra spirito nazionale e identità locali
}

\author{
AndRea Ceci \\ Università di Pisa - andrea.ceci@phd.unipi.it
}

Il saggio intende analizzare la produzione statuaria dedicata ai protagonisti della stagione risorgimentale in Emilia-Romagna e il suo ruolo nella costituzione di un'identità nazionale. Le statue e i busti dedicati ai padri della patria e ai patrioti consentono di mettere in luce le peculiarità regionali del processo di nazionalizzazione. In quest'ottica, l'articolo esplora le interazioni e le iniziative sorte dal dialogo tra storia locale e retorica nazionale.

Parole CHIAVE: Risorgimento, monUmenti, EMILIA-Romagna, StATUE, IDENTITÀ

Itinerary among the Risorgimento Monuments in Emilia-Romagna between National Spirit and Local Identities.

The essay analyses the statuary production representing the protagonists of the Italian Risorgimento in Emilia-Romagna and its role in the development of national identity. The statues and the busts dedicated to the Italian fathers of the nation and patriots allow us to highlight the regional peculiarities of the nation-building process. From this perspective, the article explores which interactions and initiatives have been affected by the dialogue between local history and national rhetoric.

KEYWORDS: ITALIAN RISORGIMENTO, MONUMENTS, EMILIA-ROMAGNA, STATUES, IDENTITY

\section{Premessa}

In uno scritto apparso in occasione del $150^{\circ}$ anniversario dell'Unità d'Italia, Roberto Balzani invitava a riflettere su una peculiare caratteristica identitaria dell’Emilia-Romagna, troppo spesso ignorata: l'essere frutto come poche altre realtà del processo risorgimentale. Prima del 1859, l’ipotesi di modificare gli assetti politico-territoriali degli Stati preunitari italiani del centro non sembrava praticabile al concerto delle potenze europee. Ancora trent'anni più tardi, il senatore Antonio Montanari (1811-1898) dichiarava: «se i morti potessero alzare il capo dalla tomba, e vedere ora l'Italia [...], certo ai morti parrebbe un sogno, come ora ai vecchi appare» ${ }^{1}$. L'inaspettato svolgersi degli eventi è denunciato anche dalla

1 Per l'inaugurazione del busto al Re Vittorio Emanuele II nella sala comunale di Meldola il giorno 23 giugno 1889 furono pronunciate le seguenti parole dal sindaco Antonio Montanari, Senatore del Regno, in Documenti d'Archivio 2002, 29. 


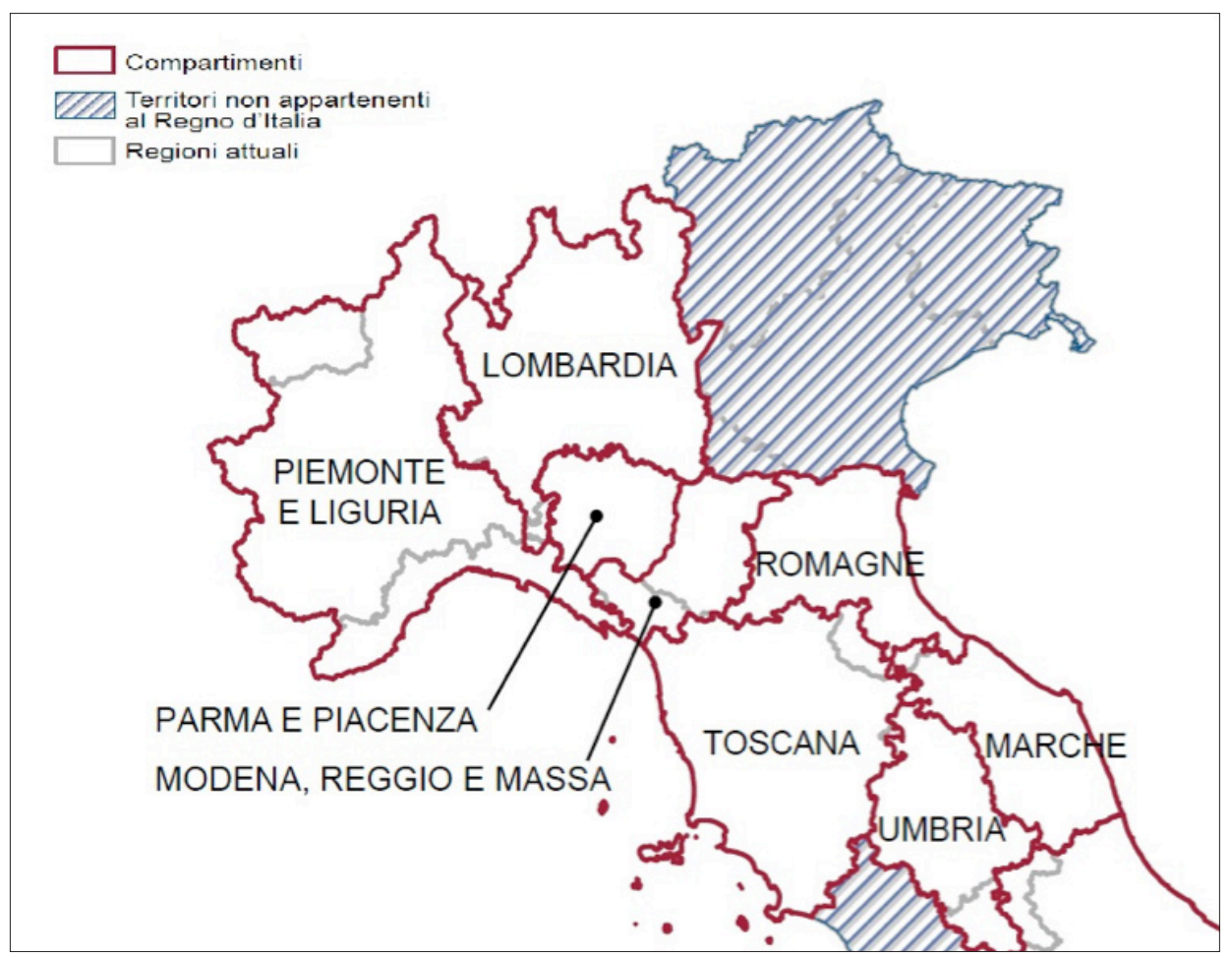

Fig. 1. Compartimenti territoriali nell'anno 1861 [Struttura e dinamica delle unità amministrative territoriali italiane 2018].

scelta di un toponimo classico per indicare la nuova entità territoriale: l'Emilia. Si rende tuttavia necessaria una precisazione: con il nuovo appellativo si intendeva una mera regione geografica comprendente i vecchi ducati, granducati e le estinte legazioni pontificie, quale «contenitore improbabile ma plausibile della composita realtà cispadana» [Balzani 2011, 18]. Il Parlamento aveva infatti osteggiato la visione regionalista sostenuta da Luigi Carlo Farini, e ripresa in seguito da Marco Minghetti, abbandonandola in favore dell'estensione alle province emiliane del regio decreto del 23 ottobre 1859, cioè il cosiddetto decreto Rattazzi, che stabiliva la suddivisione gerarchica del territorio in diversi livelli amministrativi: province, circondari, mandamenti e comuni. L'entrata in vigore della legge del 20 marzo 1865 (legge Lanza) sancì la definitiva affermazione del modello gerarchico-accentrato, a discapito di quello regionalista-decentrato, applicando uniformemente a tutto il Regno il decreto Rattazzi senza tenere conto delle peculiarità istituzionali dei singoli Stati preunitari [Rotelli 1980; Zanni Rosiello 1965]. Pertanto, nel presente saggio, il termine Emilia-Romagna verrà utilizzato soltanto per indicare l'attuale ambito territoriale della Regione, nata con le elezioni del 7 giugno 1970. Anche se non dal punto di vista amministrativo, lo strutturarsi della nazione coincise dunque con la formazione della regione e i «processi identitari e culturali, imperniati sulla memoria collettiva, di territori piccoli e grandi, finirono per fare 


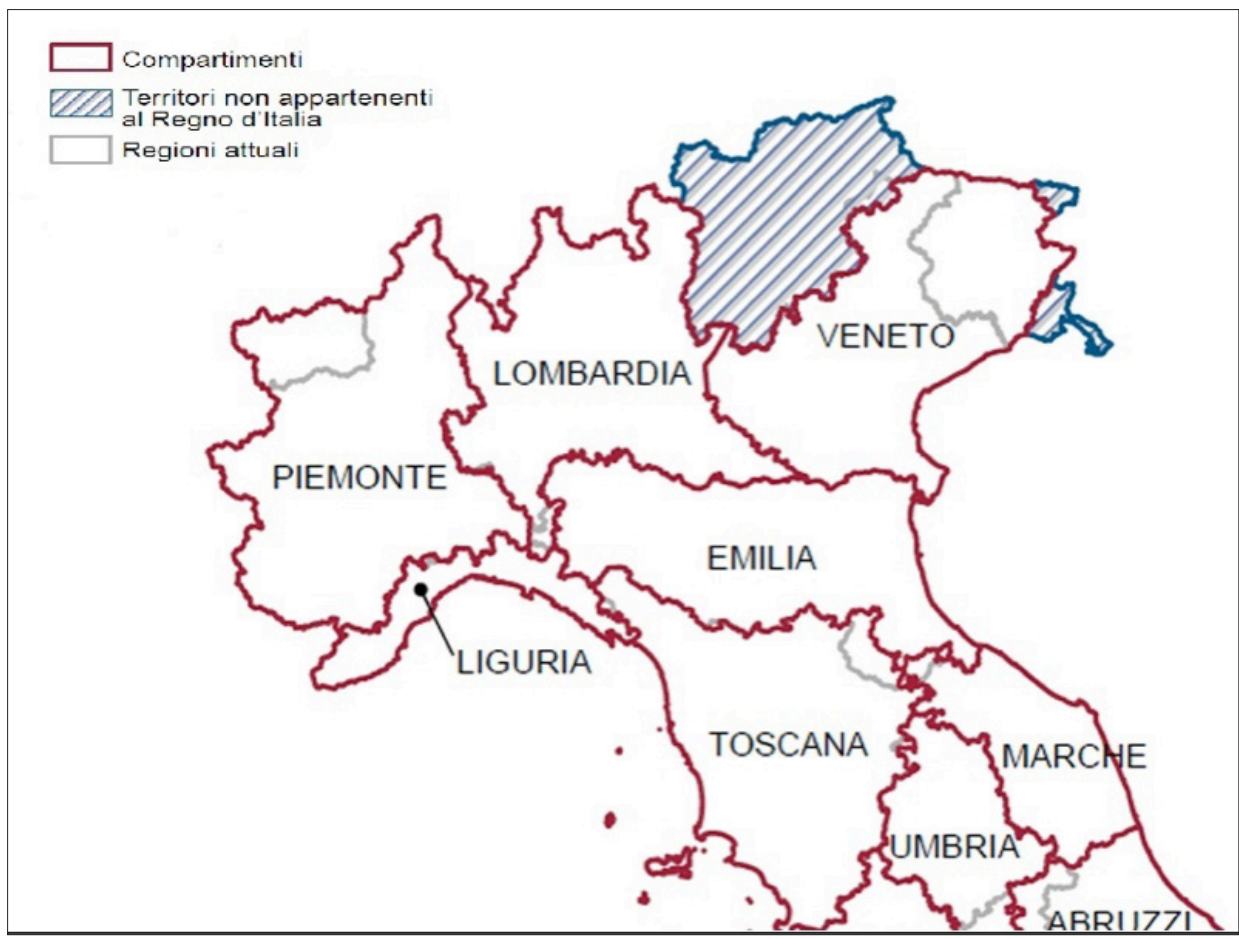

Fig. 2. Compartimenti territoriali nell'anno 1871 [Struttura e dinamica delle unità amministrative territoriali italiane 2018].

i conti con questa natura ancipite» [Balzani 2011, 18]. La necessità di creare un senso di italianità si coniugava, in misura maggiore che in altre zone della penisola, alla necessità di creare un legame regionale, superando i campanilismi, e la memoria del Risorgimento rispondeva all'esigenza di un culto laico della nazione teso a dare un senso di passato condiviso e di identità presente [Körner, Riall 2009]. Si trattava in ogni caso di una «nazione immaginata», ancora da formarsi, che trovava la sua messa in scena nello spazio urbano, attraverso la partecipazione popolare alle commemorazioni, agli anniversari, alle ricorrenze centenarie o cinquantenarie, alle cerimonie di inaugurazione dei monumenti o, ancora, alla mutazione della toponomastica cittadina [Isnenghi 1989; Isnenghi 1996-1997; Levra 1992; Tobia 1991]. In questo contesto, a partire dagli anni Ottanta del XIX secolo, le amministrazioni pubbliche si abbandonarono a un'impetuosa smania monumentalistica, che non di rado prese le forme di una vera e propria «statuomanie» [Agulhon 1988; Corgnati, Mellini, Poli 1990].

Questo contributo intende esaminare la produzione statuaria a tema risorgimentale in Emilia-Romagna, cercando di desumere i caratteri che connotarono il processo di memorializzazione della nascita della nazione. Oltre agli onori resi agli eroi patri, le élites municipali mostrarono la volontà di commemorare le celebrità locali che avevano avuto un ruolo nel processo di indipendenza, rimarcando lo sforzo patriottico degli uomini del territorio per raggiungere l'obiettivo comune. 
Inoltre, si cercherà di analizzare il conflitto tra narrazioni locali ed epica nazionale del processo unitario anche in tempi recenti. In particolare, verrà esaminata la polemica antirisorgimentale intorno alla figura del generale Enrico Cialdini, di cui alcuni vorrebbero una damnatio memoriae e che trova espressione nella contestazione agli omaggi attribuitigli, risemantizzati alla luce di problematiche attuali.

Come riferimento di base per questa indagine sarà utilizzato il volume Monumenti Tricolori, opportunamente integrato con la banca dati disponibile sul sito dedicato al Patrimonio culturale dell'Emilia-Romagna². L'opera curata da Orlando Piraccini ha il merito di aver schedato un repertorio ricco e diffuso capillarmente sul territorio, come dimostrano i 34 monumenti, i 58 busti, i 4 altorilievi e gli 81 medaglioni dedicati ai padri della patria e ai patrioti, per un totale di 177 sculture a tema risorgimentale presenti in regione. Questi dati sono frutto di una selezione all'interno del catalogo che esclude le lapidi epigrafiche, sebbene ne analizzi i medaglioni posti a decorazione, in modo da poter rendere conto degli omaggi tributati ai protagonisti del Risorgimento italiano anche da parte di quei soggetti sprovvisti dei grandi quantitativi di denaro richiesti per l'erezione di un monumento: piccole amministrazioni comunali, associazioni e organizzazioni politiche. Questo criterio può apparire opinabile e soggettivo, ma cerca di rendere conto della funzione della statuaria come vettore della costruzione del sentimento nazionale intorno alle grandi esperienze collettive, narrazione di pietra, o bronzo, che prescinde dalla parola scritta, ma conserva la capacità di costruire una sua retorica.

\section{II mito di Garibaldi}

La congiuntura politica degli anni Ottanta del XIX secolo, segnata dalla crisi economica e dall'ascesa della Sinistra storica, coincise con le sempre più frequenti dipartite dei protagonisti della stagione risorgimentale, rendendo necessario un ulteriore sforzo nel processo di nazionalizzazione degli italiani rispetto ai decenni precedenti. Il senso di smarrimento per la perdita dei padri della patria venne subitamente convertito nella loro «sacralizzazione laica», rimaneggiando la memoria recente al fine di accentuarne gli aspetti di coesione sociale e portando a modello per le giovani generazioni l'abnegazione e il sacrificio dei suoi protagonisti [Baioni 2012]. Questa interpretazione del Risorgimento, che cancellava i vecchi attriti in favore del risultato unitario, trovò modo di esprimersi già all'indomani della morte di Vittorio Emanuele II (9 gennaio 1878), ma giunse al suo apice dopo la scomparsa di Giuseppe Garibaldi (2 giugno 1882).

Paradossalmente, fu proprio la dipartita di Vittorio Emanuele II, che in vita si era sempre rifiutato «di identificare la propria Casa con la Nazione appena sor- 
ta» [Mazzonis 2003, 150], a inaugurare la nuova funzione della memoria risorgimentale quale fonte di legittimazione politica e simbolica delle giovani istituzioni nazionali e asse portante della pedagogia patriottica. Come faceva notare Cesare Correnti a Francesco Crispi, le esequie del monarca necessitavano di una ritualità, di cui la nazione era ancora sprovvista - «Ricordatevi che i prelati queste cose le sapevano fare bene. Non abbiamo precedenti. Inventateli» ${ }^{3}-$, ma, sotto l'occhio vigile del ministro dell' Interno, il doppio funerale del monarca fu trasformato in una manifestazione di popolo, la sua tomba al Pantheon divenne meta di pellegrinaggio nazionale e il Vittoriano romano, dedicato alla sua memoria, assurse ad altare della patria, identificando il sovrano con l'unica istituzione capace di rappresentare l'unità della nazione [Tobia 1991; Levra 1992; Tobia 1995]. Nonostante la monarchia restasse al centro dell'universo simbolico istituzionale, Crispi fu capace d'integrarvi la figura di Garibaldi, garantendo alla Real casa una maggiore legittimazione dal basso e opponendo «la barriera della storia e delle sacre memorie patrie recenti alla delegittimazione messa in avanti dai ceti popolari in movimento e dai loro portavoce politici» [Levra 1992, 353]. Sfruttando la predisposizione del personaggio a colpire la fantasia delle masse, dimostrata dalla pubblicazione di ben quattro biografie nel quinquennio successivo alla sua morte [Dumas 1882; Guerzoni 1882; Bizzoni 1883; Mario 1887], e ritenute ormai innocue le sue tendenze sovversive dai monarchici, Crispi cercò di riappropriarsi della figura dell'“eroe dei due mondi” in una dimensione esclusivamente nazionale e patriottica, sottraendolo al monopolio dell'iconografia democratica e innestando intorno alla sua figura una ripresa dello sforzo celebrativo che coinvolse in maniera massiccia e capillare le istituzioni sul territorio.

Per quanto rasenti l'ovvietà, per affermarsi e durare, la memoria collettiva necessita di gruppi concreti di uomini che vi si identifichino e se ne facciano portatori [Halbwachs 2001]. Pertanto, la pedagogia patriottica assunse caratteristiche differenti in relazione alle realtà territoriali e ai suoi gradi di assimilazione, o resistenza, da parte delle popolazioni. La storiografia ha individuato nell'approccio territoriale lo strumento più adatto a questo tipo di indagini, rilevando una pluralità di miti del Risorgimento e di bilanciamenti tra senso di appartenenza nazionale e identità locali [Morandi 2009; Parker 2011; Galimi 2015]. L'attenzione alle singole realtà, sia a livello cittadino sia a livello provinciale, è risultato particolarmente fruttuoso per studiare il caso emiliano-romagnolo [Porciani 1993; Körner 2009; Baioni 2010], ancora influenzato dal suo assetto preunitario particolarmente parcellizzato ed eterogeneo. La natura composita e artificiosa dell'Emilia - «un espediente politico anzi che un organismo amministrativo» secondo le parole di Minghetti [Zanni Rosiello 2000] - era ben evidenziata da Carlo Cattaneo nella sua critica al progetto regionalista di Farini.

3 Lettera di Correnti a Crispi, gennaio 1878, in Palamenghi-Crispi 1912. 
Il ministro [dell'Interno Farini] accenna a tre gruppi distinti del Piemonte, della Liguria, della Sardegna, sebbene la Sardegna da poco più d'un secolo, e la Liguria da poco meno d'un mezzo secolo, siano ridotte a vivere, volenti o nolenti, nello Stato compatto. [...] Ma egli accenna a fare un gruppo solo di ciò che ora si chiama Emilia, benché la forma e la disciplina di Stato e ogni tradizione legislativa e amministrativa sia molto più diversa tra Parma e le Romagne che non tra Parma e la Toscana. Pare che il ministro, nuovo forse alle idee legislative, non s'avveda come qui sta tutto il nodo della questione. Nell'Emilia ci stanno innanzi tre sistemi legislativi e amministrativi, tanto fra loro diversi nel bene e nel male, quanto il secolo XIX è diverso dal medioevo. Abolirli del tutto non si può, senza distruggere in uno stesso colpo il male e il bene. Non si può lasciarli sussistere nemmeno in parte, senza conservare insieme all'esistenza del bene anche l'esistenza del male [Cattaneo 1861].

Per giunta, alla pletora di ordinamenti amministrativi, si associava una pluralità di esperienze risorgimentali difficilmente riconducibili a un'unica matrice - passanti da Piacenza “primogenita d’Italia”, con la sua legione Zanardi Landi, alle spiccate attenzioni per le sorti del Veneto tipiche del Risorgimento ferrarese ${ }^{4}-$ ma che, tuttavia, potevano affratellare territori appartenuti a diversi Stati preunitari. Tuttavia, percorrendo l'itinerario che ci porta tra i monumenti risorgimentali della regione, risulta difficile sovrastimare un tratto che accomunava tutti i territori: l'importanza della figura di Garibaldi. La sua effigie, sotto forma di monumento o busto, è presente nelle principali città - ad eccezione di Modena e Reggio Emilia - e in numerosi centri secondari. Il picco di questa produzione statuaria è da situarsi tra la morte dell'eroe e il 1895, con una ripresa delle onorificenze negli anni attigui al centenario della sua nascita (1907).

Tabella 1. Produzione scultorea dedicata a Garibaldi dal 1882 al 1912.

$\begin{array}{lccccc}\text { Provincia } & \text { Monumento } & \text { Busto } & \text { Medaglione } & \text { Altorilievo } & \text { Totale } \\ \text { Bologna } & 1 & 2 & 3 & - & 6 \\ \text { Ferrara } & - & 3 & 4 & - & 7 \\ \text { Forli-Cesena } & - & 1 & 4 & - & 5 \\ \text { Modena } & 1 & 1 & 2 & - & 4 \\ \text { Parma } & 2 & 1 & 6 & 1 & 10 \\ \text { Piacenza } & 2 & 2 & 1 & - & 5 \\ \text { Ravenna } & 1 & 2 & 5 & - & 8 \\ \text { Reggio Emilia } & 1 & 1 & 4 & 1 & 7 \\ \text { Rimini } & - & - & 2 & - & 2 \\ \text { Totale } & 8 & 13 & 31 & 2 & 54\end{array}$

Dal punto di vista morfologico, le rappresentazioni garibaldine non si discostavano molto l'una dall'altra: il generale era rappresentato in piedi - fatta eccezione 
Fig. 3. Fotografia del monumento a Giuseppe Garibaldi ai giardini pubblici Margherita di Piacenza, 1890 circa [foto conservata presso l'archivio della Galleria Tadini, Lovere (BG), fondo Giovanni Battista Zitti, MR44039a. Disponibile online: http://www. lombardiabeniculturali.it/fotografie/schede/ IMM-S0110-0000206].

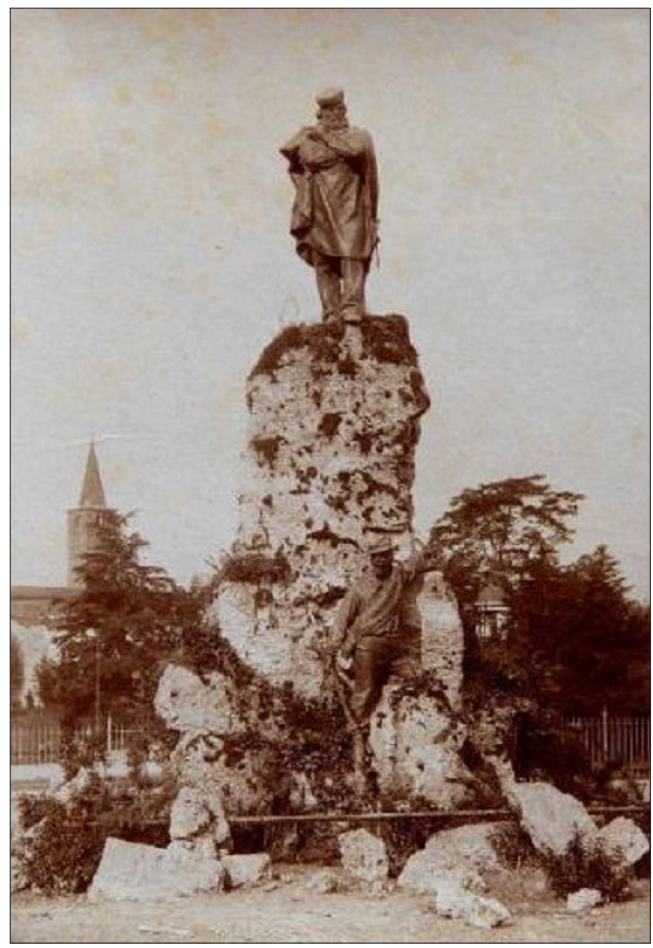

per il monumento equestre a Bologna - appoggiato alla spada, con il berretto in testa e ammantato di un poncho che lasciava intravedere la camicia rossa. Tuttavia, mentre l'iconografia di Garibaldi rimase cristallizzata nel corso degli anni, il messaggio da essa veicolato cambiò radicalmente. Infatti, tra l'Italia di Crispi e l'Italia di Giolitti si era prodotto un profondo mutamento nell'atteggiamento tenuto verso l'eroe risorgimentale e, conseguentemente, verso la funzione del monumento. In occasione del centenario della sua nascita, l'omaggio reso al generale nell'Italia giolittiana parve "più formale, solenne, ricco di ossequi devozionali, quanto meno convinto dell'attualità del mito» in un contesto pacificato, ormai sanato da tutte le precedenti fratture politiche, e garantito da uno sviluppo economico e sociale che investiva tutta la società, sebbene in maniera diseguale. Non vi era dunque più bisogno di eroi, «di martiri, di condottieri indomiti. Tempo, piuttosto, di epitaffi, quello, di memorie ritenute definitive e da consegnare alla storia, piuttosto che da far agire nelle questioni del presente» [Varni 2011, 39]. Una sola scultura in regione si discosta dal canone affermatosi in quegli anni: il Garibaldi dei giardini pubblici Margherita a Piacenza. Inaugurato nel 1889, il monumento ritraeva l'“eroe dei due mondi”, svettante su una rupe rocciosa, meditabondo e corrucciato dopo la vittoria di Bezzecca (21 luglio 1866), nel corso della Terza guerra di indipendenza. L’artista piacentino Enrico Astorri volle immortalare Garibaldi dopo essere stato costretto a pronunciare il celebre «Obbedisco!», mentre ai suoi piedi un soldato ancora ignaro dell'imminente ritirata si preparava alla bat- 
taglia, sineddoche di coloro che si schierarono al suo seguito e che vennero frenati nel loro slancio patriottico dalle scelte politiche sabaude.

Del resto, l'incompatibilità dei metodi garibaldini con le direttive governative era già stata preconizzata alcuni anni prima, quando Cavour era ancora in vita, da Giuseppe Mazzini.

Due uomini si contendono oggi i fati d'Italia: due uomini, due sistemi: Garibaldi e Cavour. [...] Sta ordinata dietro Cavour l'Italia officiale, [...] gli uomini governativi che furono, sono o sperano di essere, dei Comitati addormentatori, dei tiepidi per animo volgare o cieco intelletto. [...] Sta dietro Garibaldi l'Italia non-officiale, l'Italia popolare, l'Italia dei volontari, l'Italia dei giovani, l'Italia di quanti non guardano che al Dovere, sagrificano [sic], combattono e vincono; l'Italia che freme per l'Unità, l'Italia dell'avvenire. [...] Garibaldi è il soldato-cittadino della Patria italiana ${ }^{5}$.

Sulla scia delle parole di Mazzini e oltre il velo della retorica romantica, si sarebbe tentati di scorgere una critica al potere centrale da parte delle élites locali nella scelta del bozzetto proposto da Astorri. Tuttavia, non ci sono evidenze per sostenere questa tesi e la peculiarità del complesso monumentale si deve probabilmente imputare all'anticlassicismo dello scultore, rilevabile in altre sue opere come il busto di Mazzini posizionato originariamente in un tempietto poco distante, e dal 2007 conservato presso il Museo del Risorgimento di Piacenza. Anzi, come ha spiegato Mario Isnenghi, la rilettura di Garibaldi alla luce dell'«Obbedisco!» divenne il fulcro della nuova retorica dell'unità nazionale di matrice crispina [Isnenghi 2007; Riall 2007]. Presentato come il generale che aveva consegnato l'Italia al monarca, dimenticando il piombo dell'Aspromonte, la sua figura si faceva testimone e garante dell' «ossequio alle istituzioni e al fatto compiuto dell'egemonia monarchica». Questa necessità da parte delle élites liberali di «visualizzare ed esaltare il felice accordo tra Monarchia e Popolo e fra Patria e Rivoluzione», definito da Isnenghi come la «diarchia di bronzo», aveva lo scopo di dare un'unitaria lettura del passato e trasmettere il messaggio politico del presente [Isnenghi 1989, 332]. La manifestazione simbolica del dualismo politico che contraddistinse il processo risorgimentale acquisì, nei territori dove i partiti radicali erano più rilevanti, un carattere rivendicativo teso ad affermare la legittimità di celebrare il proprio idolo come eroe nazionale, similarmente a quanto era stato fatto alcuni anni prima alla morte del re. Nelle aree dove invece prevalevano posizioni liberali moderate, la diarchia poteva assumere un valore inverso.

Infatti, la decisione di erigere un monumento a un personaggio di rilievo nazionale, o a un eroe locale, rappresentava un momento importante per la vita cittadina che vedeva coinvolte, in diverse configurazioni, municipalità, province, associazioni e privati. Dalla creazione di un comitato incaricato della raccolta fondi all'inaugurazione del monumento, lo svolgersi della procedura era costantemente oggetto d'attenzione da parte delle gazzette locali e causa di accese discussioni nei consigli 
municipali, soprattutto nel momento in cui questi ultimi dovevano integrare i fondi raccolti dalle sottoscrizioni popolari. Le tappe principali di questo processo si possono indicare nell'identificazione del sito, nella scelta tra i bozzetti presentati dagli artisti e nella definizione delle modalità d'inaugurazione del monumento. Quest'ultima fase rappresentava spesso il momento più delicato, poiché doveva rispondere a due esigenze fondamentali: essere il più magnificente possibile e manifestare un largo consenso popolare. Nel caso in cui avesse partecipato il monarca, o qualche membro della Real casa, era necessario imprimere un carattere ancor più ufficiale e sfarzoso all'avvenimento. Le stesse narrazioni dell'evento dovevano immortalare il senso di unità e condivisione da tramandare ai posteri, come si dichiarava, nel 1886, in occasione dell'inaugurazione del monumento a Garibaldi a Finale Emilia.

Si radunavano e in bell'ordine si schieravano il lodato Comitato Esecutivo a capo del quale sta il suo Presidente onorario Onorevole Comm. Alfredo Baccarini, [...] il Sindaco con la Giunta Municipale, i Consigli Amministrativi dei locali Corpi Morali, la Società dei Reduci, le Società Operaie di Finale e Massa, la Società Ginnastica, la Società Filodrammatica, l'Associazione degli Operai Braccianti, gli Insegnanti e gli alunni delle Scuole Maschili Secondarie ed Elementari Urbane, le Autorità e i funzionari governativi quivi residenti, le Rappresentanze, con le rispettive bandiere, della Società dei Reduci di Carpi, di Modena, di Mirandola, di Bondeno, Radicali di Mirandola e Ficarolo, Circolo Federico Campanella di Ficarolo, le Società operaie di Bondeno, San Felice, San Carlo, Sant’Agostino, la Società Ginnastica del Panaro, il Club dei Reduci di Modena, Reduci di Cento. Onorano la festa molti ospiti illustri e i rappresentanti della stampa nei corrispondenti del Panaro, Giornale di Modena, della Stefani, Secolo, Democrazia, Epoca, Favilla e Naviglio. Assiste folla straordinaria di Popolo. [...] [Il Sindaco] accetta in consegna il monumento, alla cui erezione concorse la cittadinanza tutta, e fa promessa solenne che sarà gelosamente custodito perché ai più tardi nipoti ricordi le epiche gesta di Garibaldi, più grande degli Eroi di Omero, degli uomini di Plutarco. Queste parole sono salutate dai più vivi applausi ${ }^{6}$.

\section{La statuaria sabauda}

Estendendo l'analisi ai monumenti celebrativi dedicati alla componente monarchica, la volontà di rappresentare le due anime del Risorgimento risulta lampante. Esaminando i busti e i medaglioni dedicati a Vittorio Emanuele II, emerge con chiarezza come il sovrano fosse difficilmente onorato singolarmente, soprattutto nelle zone più periferiche e presso le municipalità minori. Dei sei busti a lui dedicati, due sono affiancati a Garibaldi (Imola e Meldola), mentre altri due fanno parte di un pantheon risorgimentale (Portomaggiore e Ravenna); la stessa situazione si

$6 \quad$ F. Zanasi, Verbale dell'inaugurazione del monumento a Garibaldi; Finale Emilia, 16 maggio 1886, in Finale 2008, 38-40. 


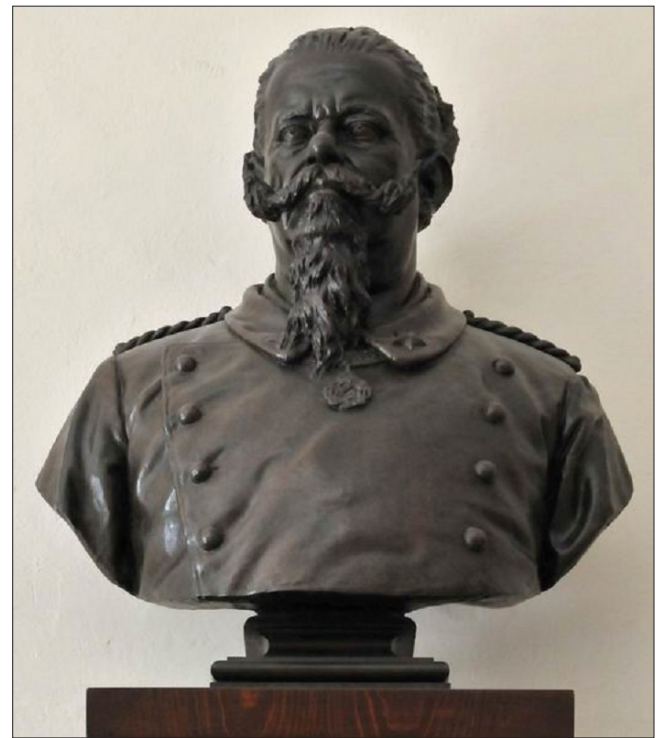

Fig. 4. Fantacchiotti C. 1888, Busto di Vittorio Emanuele II [Bronzo] Meldola, Italia: Palazzo Municipale [foto del Servizio Patrimonio Culturale dell'Emilia-Romagna. Disponibile online: https://bit.ly/3bpldzg].

presenta per sei delle dieci effigi, medaglioni o altorilievi, scolpite in suo onore. Al contrario, la sua impronta sui grandi complessi monumentali risulta più marcata. Questa tendenza ha portato una parte rilevante della storiografia a identificare una contrapposizione tra una monumentalità forzata e ufficiale dedicata al sovrano e una più spontanea e popolare dedicata a Garibaldi. Nella maggior parte dei casi, le sculture del generale prendevano avvio da un comitato, legato ad associazioni politiche, combattentistiche od operaie, che organizzava sottoscrizioni popolari, mentre la statuaria tributata alla componente sabauda traeva spesso origine da proposte avanzate dalle istituzioni. A riprova dello svolgersi di queste dinamiche, nel corso del 1888, in occasione della visita in Romagna del re Umberto I e della regina Margherita, si registrò un particolare impegno nel tributare onori al "gran re" da parte dei consigli municipali, in modo da poter inaugurare i monumenti alla presenza dei reali. In alcuni casi, come a Meldola, si arrivò a modificare addirittura il progetto originale, stimando troppo modesta una semplice lapide con gli stemmi intrecciati di Casa Savoia e del Comune, da affiancare a un busto di Garibaldi. Il busto di Vittorio Emanuele II che si decise di erigere venne inaugurato solo l'anno seguente a causa della cancellazione della visita dei sovrani. Durante la cerimonia, il sindaco Antonio Montanari, ex senatore del Regno, elogiò a gran voce l'operato benefico della monarchia, prendendo le distanze dalle posizioni radicali.

Francesco Crispi anni addietro in una lettera a Giuseppe Mazzini disse: la Monarchia ci unisce la Repubblica ci divide. Paolo Boselli nelle festività dell'Esposizione Emiliana l'anno scorso in Bologna affermava: In Italia esiste una monarchia di cui non si ricorda che sia mai esistita una Repubblica migliore. Oggi davanti all'immagine di Vittorio Emanuele sia concesso ad un vecchio Romagnolo che dalla giovanezza [sic] fino a questo dì, partecipò col cuore e coll'opera alle vicende dell'Italica Epopea, sia concesso di ricordare con affettuoso ossequio al Re Umberto, e con franca parola ai concittadini: Che la casa Sabauda fu la pietra 
angolare del grandioso edifizio, è, e deve restare il capo saldo dell'unità, dell'indipendenza, e della libertà nazionale! ${ }^{7}$

Una certa disaffezione è invece rilevabile per la figura di Camillo Benso, conte di Cavour, di gran lunga il meno onorato in regione tra gli artefici dell'Italia unita. Rappresentato in luogo di Vittorio Emanuele II per designare la componente monarchica del processo risorgimentale, a lui furono dedicati solo otto busti; di questi, la metà sono affiancati da un busto di Garibaldi (Crevalcore, Poggio Renatico, Ferrara e Montecchio Emilia), mentre due fanno parte di un pantheon (Portomaggiore e Imola). Questa disparità di attenzioni dovette essere ben percepita anche dai contemporanei se, nel 1888, gli studenti del circolo monarchico liberale bolognese ritennero necessario accompagnare il busto nei giardini della piazza a lui intitolata con l'epigrafe: «CAMILlo CAVOUR. PERChÉ QUESTA PIAZZA OLTRE IL NOME RICORDI ANCHE LE SEMBIANZE DELLO STATISTA GLORIOSO».

Alla luce di queste considerazioni, risulta complesso giudicare la genuinità delle commemorazioni e stilare un bilancio univoco, a livello regionale, del peso esercitato dall’iconografia sabauda nei processi identitari. Nella sua analisi sul ruolo della monarchia nel processo di costruzione del sentimento nazionale, Catherine Brice suggeriva che un'analisi qualitativa e quantitativa della statuaria avrebbe potuto informarci sulla consistenza del sentimento unitario colto nel suo rapporto di bilanciamento fra identità locale e identità nazionale [Brice 2004, 687; Brice 2012]. Estrapolando i dati relativi alle province emiliane e romagnole (tabella 2 e tabella 3) è stato possibile individuare tre strategie d'approccio adottate dalle amministrazioni per la commemorazione di Vittorio Emanuele II.

Tabella 2. Adesioni al lutto nazionale per Vittorio Emanuele II (1878-1879) [Brice 2004; Abeille 1879].

\begin{tabular}{lccc} 
Provincia & $\begin{array}{c}\text { NUMERO DEI COMUNI } \\
\text { ADERENTI ALLE } \\
\text { COMMEMORAZIONI PER IL } \\
\text { SOVRANO }\end{array}$ & $\begin{array}{c}\text { COMUNI ADERENTI ALLA } \\
\text { SOTTOSCRIZIONE PER IL } \\
\text { MONUMENTO NAZIONALE }\end{array}$ & $\begin{array}{c}\text { SOMME VERSATE } \\
\text { PER IL MONUMENTO } \\
\text { NAZIONALE IN LiRE }\end{array}$ \\
Bologna & 35 & 1 & 10.000 CC \\
\hline Ferrara & 15 & 5 & 1.450 \\
Forlì & 33 & 8 & 1.510 \\
Modena & 43 & 5 & 560 \\
Parma & 50 & 12 & 1.060 \\
Piacenza & 38 & 7 & 550 \\
Ravenna & 26 & 9 & 3.000 CC +4.600 \\
Reggio Emilia & 38 & 4 & 325 \\
CC= Consiglio Comunale & & &
\end{tabular}

Per l'inaugurazione del busto al Re Vittorio Emanuele II nella sala comunale di Meldola il giorno 23 giugno 1889 furono pronunciate le seguenti parole dal sindaco Antonio Montanari, Senatore del Regno, in Documenti d'Archivio 2002, 30. 


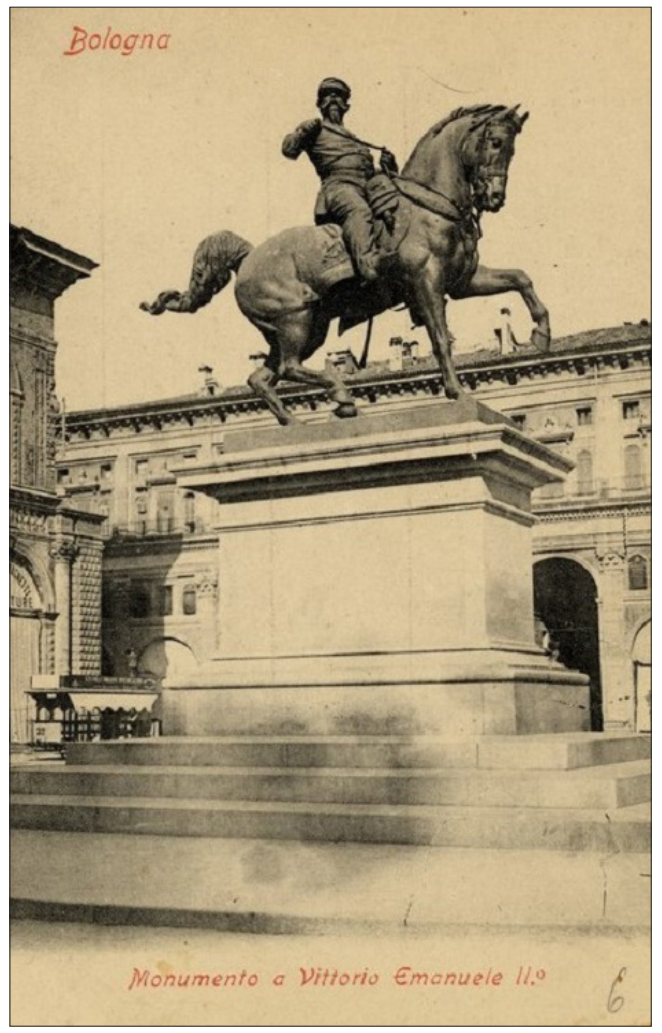

Fig. 5. Cartolina postale raffigurante il monumento equestre a Vittorio Emanuele II nella sua sede originale di piazza Maggiore a Bologna, ora ai giardini Margherita [foto conservata presso l'archivio della Biblioteca dell'Archiginnasio, Bologna, Raccolta Parmeggiani, n. 056. Disponibile online: http://badigit.comune.bologna.it/Fotografie/dettaglio.asp?lettera=51].

Tabella 3. Monumenti locali a Vittorio Emanuele II [Brice 2004; Abeille 1879].

\begin{tabular}{|c|c|c|c|}
\hline Provincia & $\begin{array}{c}\text { SITO DEL } \\
\text { MONUMENTO } \\
\text { LOCALE }\end{array}$ & $\begin{array}{l}\text { COMUNI ADERENTI ALLA } \\
\text { SOTTOSCRIZIONE PER IL } \\
\text { MONUMENTO LOCALE }\end{array}$ & $\begin{array}{c}\text { SOMME VERSATE PER II } \\
\text { MONUMENTO LOCALE } \\
\text { IN LIRE }\end{array}$ \\
\hline Bologna & Bologna & 1 & $50.000 \mathrm{GP}$ \\
\hline Ferrara & Ferrara & 4 & $10.000 \mathrm{CC}+700$ \\
\hline Forlì & Bologna & 1 & 50 \\
\hline Modena & Modena & 6 & $25.000 \mathrm{CC}+1.350$ \\
\hline$C C=$ Consiglio Comunale & $G P=$ Giunta & ale & \\
\hline
\end{tabular}

La prima, adottata dal Consiglio comunale di Ravenna, consistette nel finanziare esclusivamente il monumento nazionale a Roma. La seconda, e la più diffusa tra le realtà territoriali regionali, fu di optare per una distribuzione dei fondi, più o meno equilibrata, tra i monumenti provinciali e il monumento nazionale: ad esempio, il Consiglio comunale bolognese stanziò 10.000 lire da devolvere all'erezione del Vittoriano, mentre la Giunta provinciale versò una somma considerevole, 50.000 lire, per il monumento equestre a Vittorio Emanuele II da collocarsi nella piazza intitolata al sovrano, oggi piazza Maggiore, in occasione dell’VIII centenario dell'Università felsinea. Infine, il Consiglio comunale di Ferrara e 
quello di Modena privilegiarono un approccio localistico, finanziando esclusivamente il proprio monumento cittadino.

Nel complesso, dall'analisi dei dati non emerge una netta contrapposizione alla monarchia, che appare generalmente accettata negli anni Ottanta del XIX secolo, o una certa freddezza verso la memoria del "re galantuomo", quanto piuttosto una critica alla centralità assunta da Roma. Le somme stanziate per il monumento nazionale risultano decisamente inferiori rispetto a quelle stanziate per i monumenti locali - 24.055 lire per il primo, 87.100 lire per i secondi - sebbene alle sottoscrizioni per questi ultimi avessero aderito meno municipalità. Inoltre, molti comuni aderenti alle commemorazioni nazionali preferirono onorare Vittorio Emanuele II con lapidi cittadine, atti di carità pubblica, suffragi di messe e contributi alla costruzione di ospedali piuttosto che sostenere le spese per il monumento romano. Come menzionato, a Bologna, la Giunta provinciale deliberò di concorrere con 50.000 lire al monumento equestre di Vittorio Emanuele, stanziando una somma non molto differente da quella destinata al monumento equestre di Garibaldi 60.000 lire - al quale concorse però anche il Consiglio comunale [Gavelli 2012]. Già in questi anni, sembrava dunque essere privilegiata una dimensione locale nelle commemorazioni risorgimentali e il contrastato rapporto tra centro e periferia, non ancora manifesto sul piano politico come lo sarà sullo scorcio del secolo, lasciò un'impronta anche sulla statuaria. Anche laddove le somme erogate risultano molto esigue, come nel caso di Reggio Emilia, non si riscontra un consapevole disinteressamento nell'erigere il monumento, ma emergono problematiche di tipo finanziario [Carrattieri 2012].

\section{Le rappresentazioni dei patrioti tra spirito unitario e identità locali}

A partire dal 1890, sulla scia di un trend nazionale, si iniziò a registrare in ambito regionale un graduale declino della produzione statuaria (grafico 1). Analizzando i soggetti rappresentati si rileva una sostanziale cessazione della rappresentazione della componente monarchica mentre, al contrario, aumentano le rappresentazioni dei patrioti locali, accompagnate dal mai dimenticato Garibaldi. Secondo Carlo Marochetti, autore della statua di Carlo Alberto a Torino (1861), la ragione andava ricercata nello scarso effetto scenico che si otteneva nel ritrarre qualsivoglia Savoia che non fosse Vittorio Emanuele II.

Combien j'eusse préféré exécuter la statue du roi Victor-Emmanuel! Certes il n'est pas beau, notre souverain; mais, d'après lui, on pourrait faire une œuvre saisissante, originale; il a je ne sais quoi de sauvage, de pittoresque, qui ne manque pas de grandeur et rappelle un roi hun, un chef barbare [d'Ideville 1878, 49-50].

Più prosaicamente, la progressiva diminuzione dei monumenti celebrativi denota una modificata sensibilità che non identificava più nella statua il miglior tributo alla memoria del sovrano, come dimostra la scarsità di rappresentazioni di Um- 


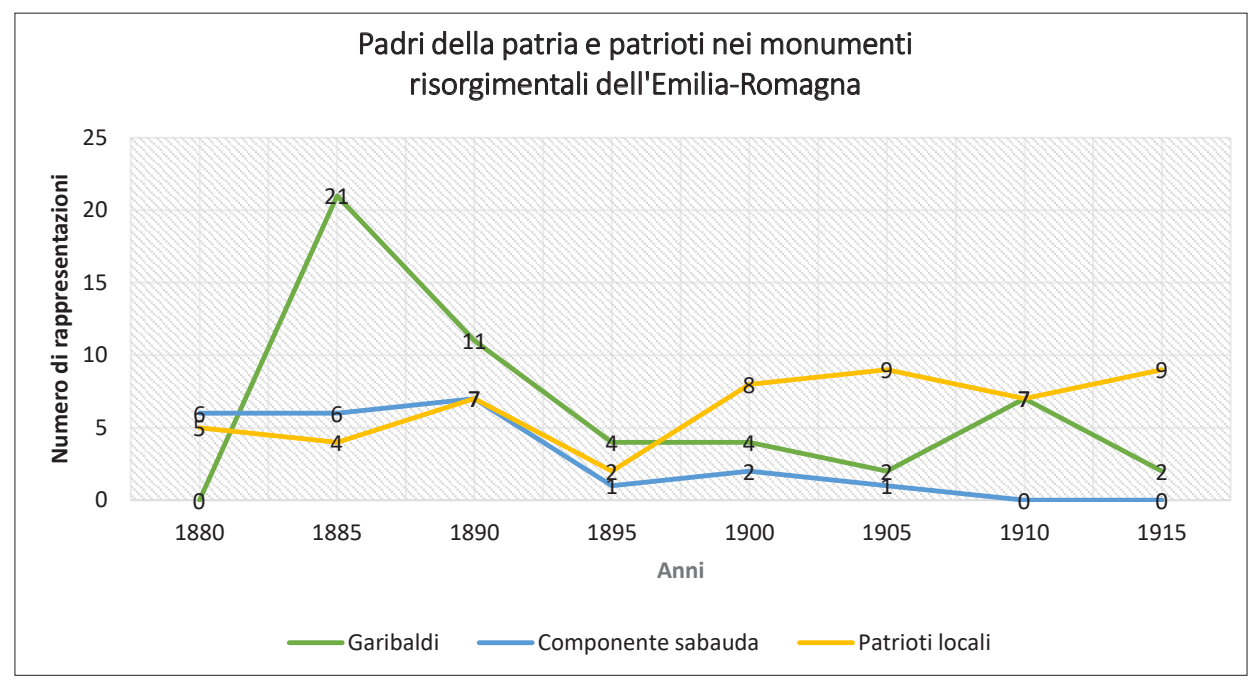

Grafico 1. Padri della patria e patrioti locali nei monumenti risorgimentali tra il 1880 e il 1915.

berto I all'indomani del suo assassinio, senza dimenticare che erano ancora in corso i lavori per il Vittoriano (inaugurato nel 1911).

Inoltre, il rafforzamento dell'estrema sinistra, tramite l'espansione delle nuove organizzazioni operaie e contadine, e la conseguente differenziazione ideologica e organizzativa tra i gruppi socialisti, repubblicani e radicali, consentì al partito socialista di affermarsi «come la maggior forza politica emiliana in tutta la fascia di pianura dal parmense alle bonifiche ferraresi; mentre il partito repubblicano prevalse in Romagna» [Lotti 1980, 307]. Con la conseguente entrata delle opposizioni socialiste e repubblicane nei consigli municipali, si iniziò a ravvisare una crescente contrarietà allo stanziamento dei fondi per la commemorazione della Real casa, che andò di pari passo con la promozione di un soggetto a loro ideologicamente più vicino. A Cesena, dopo la vittoria delle elezioni comunali da parte della compagine repubblicana, i tributi agli eroi risorgimentali ripresero con rinnovato vigore, e particolari contrasti suscitò la decisione di dedicare un busto a Eugenio Valzania (1821-1889), irriducibile patriota garibaldino ed esponente di spicco del repubblicanesimo cesenate.

Ebbe nella guerra lombarda del 1848 / con la prima ferita / il grado di capitano sul campo, / poi esule / due condanne di morte / dal papa e dall'Austria: / combatté nel 1859 e 1860 / dovunque fu levata la bandiera d'Italia: / nel 1866 a Bezzecca / aiutante del Generale / fu decorato per valor militare: / nel 1867 espugnò Monterotondo. / Delle persecuzioni e dei dolori / lo confortarono / l'amicizia di G. Mazzini e G. Garibaldi [Carducci 1938].

Tuttavia, nel corso della sua vita, Valzania aveva attirato su di sé le ostilità degli ambienti filomonarchici in seguito alle accuse che lo avevano identificato come mandante di alcuni atti violenti a scopo politico e per le sue note relazioni con l'a- 


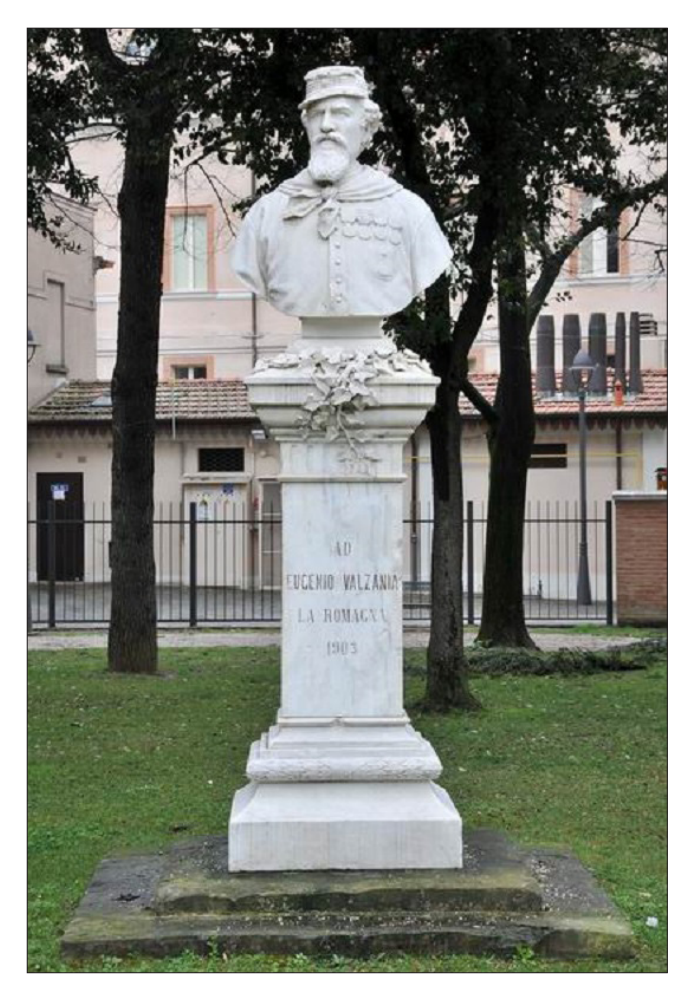

Fig. 6. Grilli P. 1903, Busto di Eugenio Valzania [Marmo] Cesena, Italia: giardino pubblico [foto del Servizio Patrimonio Culturale dell'Emilia-Romagna. Disponibile online: https://bit.ly/2OAI7e6].

narchico Celso Ceretti. Puntando proprio sulla vicinanza agli ambienti anarchici, il 2 agosto 1874, egli, insieme a molti altri esponenti mazziniani e repubblicani, tra cui Aurelio Saffi, fu fatto arrestare a Villa Ruffi, a Covignano di Rimini, con l'accusa di cospirare contro la monarchia. Due anni prima della morte la sua fede repubblicana restava incrollabile: eletto alla Camera dei deputati nel collegio di Forlì (1887), rinunciò al seggio, rifiutandosi di giurare fedeltà al re. Così, quando il 6 settembre 1903, alla presenza di un nutrito gruppo di garibaldini provenienti da ogni parte della Romagna, venne inaugurato il monumento, in molti disertarono i festeggiamenti. Se l'assenza dei monarchici non risultò essere sorprendente, lo stesso non si poté dire di quella della locale Società dei reduci delle patrie battaglie, che lo stesso Valzania aveva contribuito a fondare.

Le vicissitudini del busto cesenate di Valzania illustrano chiaramente come l'erezione di un monumento avesse la capacità di cristallizzare il dibattito politico e di come, occasionalmente, la sua inaugurazione potesse fungere da "scena" per mettere in evidenza i contrasti politici tra i differenti gruppi locali. Se a Cesena furono i filomonarchici a sfilarsi dalle celebrazioni cittadine, in altre occasioni, furono i membri dei partiti radicali a prendere le distanze, come avvenne all'inaugurazione del monumento ai Caduti per l'indipendenza e per la libertà d'Italia a Ravenna. Composta da due figure colossali, Ravenna e un soldato in atto di spirare sul campo, l'opera di Cesare Zocchi (1851-1922) è riconosciuta come uno dei più begli esempi della statuaria risorgimentale. Ravenna è allegoricamente rappresentata in figura di donna guerriera gota e incoronata di querce, mentre, 


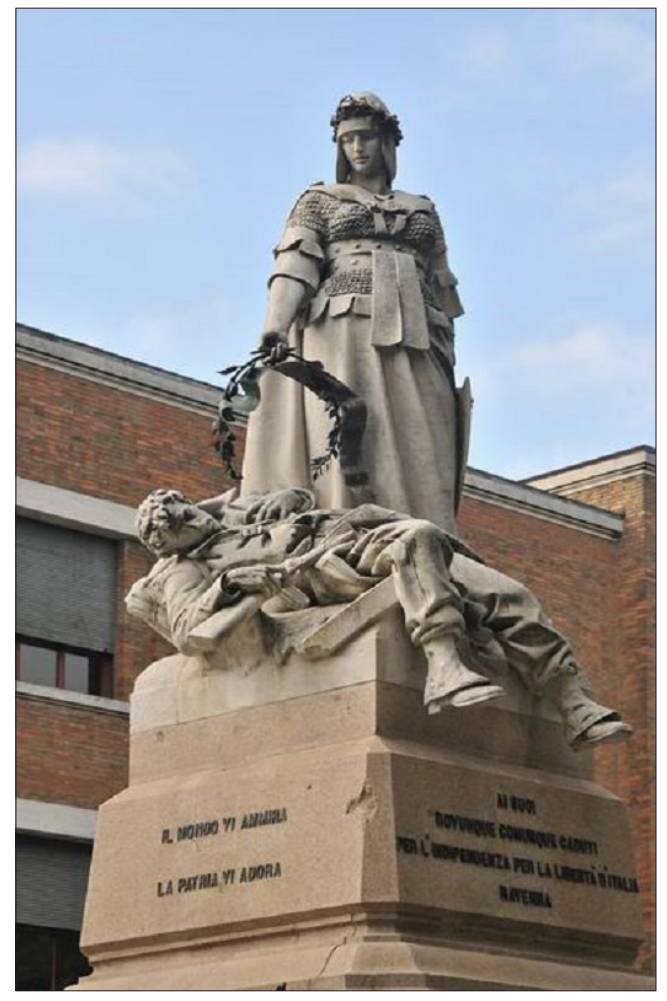

Fig. 7. Zocchi C. 1888, Monumento ai Caduti per l'indipendenza e per la libertà d'Italia [Marmo con bassorilievi bronzei] Ravenna, Italia: giardini di piazza Anita Garibaldi [foto del Servizio Patrimonio Culturale dell'Emilia-Romagna. Disponibile online: https://bit.ly/30EFDyl].

con la mano destra, depone una corona d'alloro sul soldato che le giace ai piedi, e con la sinistra tiene alzato uno scudo con il blasone ravennate. Una peculiarità del monumento è la scelta di ornare la base con un bassorilievo raffigurante sul lato sud Anita Garibaldi a cavallo, e sul lato nord Giuseppe Garibaldi dolente di fronte al suo corpo esanime. La statuaria pubblica del XIX secolo era infatti di genere maschile e assumeva attributi guerreschi, rappresentati spesso dalla spada, dall'uniforme e dal cavallo; solitamente, l'unica figura femminile rappresentata era, appunto, l'allegoria della patria, e si dovranno attendere gli anni Ottanta del $\mathrm{XX}$ secolo perché Anita venga onorata di un monumento a lei specificatamente dedicato alla cascina delle Mandriole (1985).

La causa delle tensioni di fronte a un monumento percepito come proprio da tutte le compagini politiche fu la decisione dell'amministrazione ravennate di inaugurarlo al cospetto del re Umberto I nel corso della sua visita del 1888. Come avevano mostrato i disordini a Forlì del 1884, in occasione dell'inaugurazione del busto a Garibaldi, la Romagna era una terra per nulla soddisfatta della monarchia. La presenza del re alla cerimonia inaugurale del $1^{\circ}$ settembre poteva causare gravi disordini e quindi, al fine di garantire un'accoglienza favorevole ai reali, nel luglio 1888, Crispi decise di concedere l'amnistia all'agitatore politico Amilcare Cipriani, in carcere per omicidio e diserzione, e invano eletto deputato a Forlì e Ravenna. 
Tuttavia, venute a conoscenza dell'iniziativa del Consiglio comunale, 28 associazioni socialiste e 23 associazioni repubblicane si ritrovarono la sera del 2 agosto per stilare un documento di protesta.

Considerato che oltre ad un significato eminentemente politico, la visita delle Loro Maestà è anche destinata a portare non solo danno, ma anche completa rovina alle già troppo esauste pubbliche finanze; [...]

Considerando che codesta visita è dovuta all'invito arbitrario di pochi individui, e più specialmente al R. Sindaco [Ugo Burnazzi]. il quale ha operato in modo da porsi in perfetta contraddizione colle tendenze ed aspirazioni del Popolo romagnolo;

protestano

contro ogni atto emanato e che emanerà dal Sindaco o da qualsiasi altro rappresentante l'autorità pubblica, lasciando che la responsabilità di qualunque avvenimento originato da manifestazioni politiche in conflitto fra loro per la visita dei Reali, ricada su chi le ha promosse, e tutt’ora la fomenta e la vuole ${ }^{8}$.

Per evitare la sommossa, Aurelio Saffi scrisse una missiva al comitato direttivo della Consociazione repubblicana romagnola, sostenendo che sarebbe stata una stoltezza «gratificare i più malevoli e sleali detrattori [...], dando loro il desiderato pretesto di gridarci in massa dissennati e selvaggi e di predicare la necessità di un regime eccezionale per le nostre province» ${ }^{9}$ [Saffi 1888]. Anche Umberto I tentò di non surriscaldare gli animi, rifiutando la guardia d'onore offertagli dai veterani di Ravenna del 1848-1849, scrivendo che si riteneva «abbastanza guardato da tutto il popolo nel quale aveva pienissima fede» [Miserocchi 2014, 67]. Anche se non si giunse all'aperta insurrezione, due mesi dopo, il 4 novembre, si assistette ad una spettacolarizzazione dello scontro politico, quando socialisti e repubblicani decisero di inaugurare nuovamente il monumento, invitando come oratore Andrea Costa, primo deputato socialista del Regno d'Italia. Si trattava di una vera e propria riconsacrazione popolare, che si chiuse con un tratto di colore. «Fu presentato all'ufficio telegrafico un telegramma che diceva: disinfettato Monumento [...]; al visto del Prefetto questi vi appose: trattenuto per ragioni igieniche» [Miserocchi 2014, 70].

Dunque, l'analisi della statuaria ad uso pubblico non rileva solo problematiche relative alla costruzione di una memoria collettiva, ma anche di tipo politico. Oltre all'onore portato alla propria realtà locale, le élites liberali si dedicavano ai monumenti commemorativi poiché vi vedevano la possibilità di partecipare a

8 Documento col quale alcune frazioni della democrazia iniziarono un'agitazione, esplicata in diversi modi, per tentare di impedire la venuta dei Reali nella nostra regione, 6 agosto 1888, in Miserocchi 2014, 65.

9 Aurelio Saffi, Lettera scritta da Forlì ai Membri del Comitato Direttivo della Consociazione Repubblicana Romagnola, in Miserocchi 2014. 
un movimento nazionale, acquisendo una legittimità che, al di là del proprio territorio, risultava ancora vacillante. La comunità d'appartenenza era infatti tradizionalmente la fonte attraverso cui far scaturire le identità collettive e una risorsa strategica importante da giocare nei confronti con il potere centrale. In ultima analisi, la statua aveva la funzione di fondere la «piccola Patria» con la «Grande Patria» attraverso le azioni dell'eroe locale all'interno del più vasto arazzo della storia nazionale [Romanelli 1989]. Infatti, seppur ostili al sistema monarchico, repubblicani e socialisti rivendicavano una forte continuità con il Risorgimento, sostenendo la necessità di democratizzare gli enti locali, garantendone l'autonomia contro i tentativi di accentramento. La conquista del Comune era considerata uno dei punti cardine del programma politico, il mattone fondamentale per poter sperimentare «una democrazia politica dal basso [...], pur nel contesto di un'architettura istituzionale di stampo liberal-nazionale» [Varni 1997, 574].

L'armonizzazione di questo processo era affidata al culto laico garibaldino capace di creare «una cornice sentimentale e culturale che la sensibilità romantica avrebbe giudicato come l'espressione più genuina dell'identità democratica» [Varni 1997, 562]. Sfruttando questo sentimento largamente condiviso, e appoggiandosi al diffuso associazionismo, le giunte radical-repubblicane occuparono simbolicamente i luoghi centrali dei paesi, sottraendoli alla Chiesa - che si oppose fortemente alla sacralizzazione dell'identità nazionale, ma da cui vennero prese in prestito le liturgie patriottiche [Banti 2000] -, inventando «identità collettive che univano al rispetto del genius loci [...] una forte impronta politico ideologica» [Balzani 1997, 608]. Risulta allora evidente l'importanza per ogni paese di ricordare il proprio patriota, i propri combattenti, i propri caduti e i propri rappresentanti nei primi governi dell’'Italia unita.

Eugenio Valzania a Cesena, Luigi Carlo Farini a Ravenna e a Russi, Aurelio Saffi a Forlì, Luigi Orsini a Meldola, Don Giovanni Verità a Modigliana, Raffaele Pasi a Faenza, Ugo Bassi e Marco Minghetti a Bologna, Quirico Filopanti a Budrio, Ciro Menotti e Nicola Fabrizi a Modena, Enrico Cialdini a Castelvetro e a Reggio Emilia, Manfredo Fanti a Carpi, Antonio Panizzi a Brescello, Don Giuseppe Andreoli a Rubiera, Gian Domenico Romagnosi a Salsomaggiore e Piacenza, Angelo Balestrieri a Polesine Parmense, senza dimenticare la grande scenografia verdiana di Parma [Piraccini 2012, 18].

Soprattutto in Romagna, dove, nel corso del primo decennio unitario, l'associazionismo era stato fortemente perseguitato dalla Destra storica, si registrò una precoce radicalizzazione delle elezioni e, di conseguenza, la statuaria divenne un ulteriore fronte della battaglia politica. Negli ex ducati, dove le gerarchie del notabilato erano più strutturate, l'opposizione alla monarchia non raggiunse picchi di contrasto così elevati, sebbene l'elemento socialista risultasse ben attivo. Questa fase più localistica della statuaria risorgimentale si estese fino all'inizio del XX secolo quando, mentre ancora si erigevano le ultime statue ai patrioti, già si approssimava la Prima guerra mondiale, causa di una seconda grande ondata di monumenti commemorativi, di cui il milite ignoto è espressione precipua. 


\section{II ruolo della statuaria risorgimentale nell'Italia di oggi}

La storiografia ha ormai raggiunto risultati consolidati per quanto concerne l'uso della memoria risorgimentale nel corso del XX secolo [Il mito del Risorgimento 1995]. Molto si è scritto sull'appropriazione di Garibaldi da parte del regime fascista, accostando la camicia rossa a quella nera, con l'impegno di difendere la nazione sia dalle potenze straniere che dai nemici interni [Baioni 2006; Baioni 2009; Rossi 2010]. Meno compatto, invece il fronte della sinistra dove, a fianco della polemica antirisorgimentale di Togliatti, parte del Partito comunista italiano si fece portatore dei valori dell'opera di Garibaldi e dei patrioti. Il recupero della tradizione risorgimentale passò poi nel campo della Resistenza, riproponendo il tema del Risorgimento tradito, che non volgeva però lo sguardo al passato - si era ormai consumata la rottura definitiva con l'istituzione monarchica - ma che si andava facendo secondo le tradite idealità repubblicane. Nell'edizione emilianoromagnola della versione clandestina di «Noi donne» del 10 luglio 1944, si poteva leggere:

Come il leggendario Eroe del Risorgimento, i nostri giovani delle Brigate Garibaldi, con armamento leggero, combattono valorosamente su per gli ardui sentieri della montagna, contro i tedeschi e contro i fascisti. Questi giovani sono i volontari della Libertà. Essi combattono questa strenua lotta con indicibili sacrifici per l'unico grande fine: l'indipendenza della Patria, il loro motto è “Rendere l'Italia libera o morire" [Varni 1995, 542].

Dunque, la riscoperta del valore della patria e della nazione, declinate in ottica antifascista, sostennero anche una retorica che equiparava i partigiani a novelli garibaldini, traendo dall'esempio risorgimentale un'identità capace di «diffondere una possibilità di speranza di fronte al disgregarsi della collettività nazionale e battersi per ritrovarne le ragioni del cammino verso l'avvenire» [Varni 2011, 46]. La nuova Italia da costruire, sebbene trovasse corpo e ispirazione in Garibaldi, doveva molto all'opera di Mazzini, alle sue speranze di rinnovamento globale realizzate attraverso una massiccia partecipazione popolare. E nel secondo dopoguerra sarà proprio la figura di Mazzini, ultimo ad entrare nel pantheon d'Italia, a ricevere i maggiori onori. Un culto mazziniano aveva preso piede ancora prima della sua morte (10 marzo 1872) - all'indomani della quale si cercò di mummificare il suo corpo per farne una statua corporea [Luzzatto 2001] -, trovando tuttavia un'espressione pubblica ufficiale soltanto durante il periodo crispino, e compiendosi completamente nell'Italia giolittiana quando ormai non era più il repubblicanesimo a suscitare le maggiori preoccupazioni delle istituzioni, ma il movimento socialista. L'elevazione del pensatore genovese a padre della patria aveva l'obiettivo di integrare una parte selezionata e depurata dell'universo simbolico della compagine repubblicana nell'ufficialità del Regno, disinnescando ulteriori possibili contrasti che avrebbero minato la stabilità sociale. In quest'ottica vanno intesi il disegno di legge presentato da Crispi nel 1892 per l'erezione di un monumento nazionale da erigere a Roma, l'introduzione dei Doveri dell'uo- 
mo - purgato dalle parti relative alla monarchia - come libro di testo nelle scuole (1901) e le onoranze ufficiali del 1905 in occasione del centenario della sua nascita. Tuttavia, l'eredità di Mazzini non divenne mai un effettivo elemento di unificazione per le culture politiche italiane [Della Peruta 1995; Levis Sullam 2010]. In alcune città, il culto laico mazziniano trovò modo di esprimersi già prima della rivalutazione istituzionale con l'allestimento dei busti di Portomaggiore (1882-84), Parma (1887), Piacenza (1889) e Forlì (1890), ma, a causa del diffondersi dell'ideologia socialista in Emilia, il numero di rappresentazioni restò esiguo negli anni compresi tra la sua entrata nel pantheon d'Italia e lo scoppio della Prima guerra mondiale. Fu nel secondo dopoguerra che in Romagna, e soprattutto nel Ravennate, dove era ben radicato il Partito repubblicano italiano [Cavazza, Berardi 1980; Ridolfi 1989], si registrò un moltiplicarsi di busti in suo onore. In tempi più recenti, fu invece il suo ruolo di precursore dell'Europa unita a essere enfatizzato, come a Salsomaggiore Terme di Parma: Giuseppe / MazzinI / Precursore / DEGLI / STATI / UnITI / D’EUROPA.

La lettura del Risorgimento di matrice crispina, e i suoi effetti, si sono quindi innestati nella storia dell'Italia repubblicana, divenendo un mito fondativo dal quale hanno attinto le classi dirigenti per accreditarsi nell'agone politico. Tuttavia, ciò non ha portato alla pacificazione del corpo sociale auspicata. Sul finire del XX secolo, il Risorgimento è divenuto bersaglio di attacchi e critiche sia da parte degli ambienti vicini all'integralismo cattolico sia da parte dei movimenti autonomistici. Negli anni Novanta, con evidente riferimento al partito politico della Lega Nord, Giovanni Spadolini commentava:

Oggi il Risorgimento è contestato e discusso da tante parti. Si cercano le identità regionali contro i requisiti della prevalente identità nazionale. Si confonde il moto unitario con una specie di guerra civile ancora non ricomposta. Si rischia anche di compromettere o appannare le fondamenta di legittimità di questa Repubblica, la Repubblica nata dall'intuizione mazziniana della Costituente popolare, proprio congiungendo i valori del primo e secondo Risorgimento [Spadolini 1995, 5].

Più recentemente, si è registrata una significativa riproposizione di tesi neoborboniche nel Sud che ha trovato nuovo vigore proprio in occasione del $150^{\circ}$ anniversario dell'Unità d'Italia. Nel proliferare di volumi dedicati alla ricostruzione dell'epopea risorgimentale apparsi tra la fine del 2010 e l'inizio del 2011, hanno attirato una particolare attenzione da parte dell'opinione pubblica i titoli che esprimevano critiche severe nei confronti del Risorgimento, rimettendo in discussione i vantaggi del processo unitario e negandone le motivazioni ideali ${ }^{10}$. Tra i temi più visitati da questa pubblicistica, che spesso attinge a piene mani da tesi legittimiste e filoclericali del XIX secolo, vi sono la denuncia della spoliazione nei confronti della Chiesa e la rivalutazione del brigantaggio come movimento di

10 Per una panoramica delle pubblicazioni in occasione del $150^{\circ}$ anniversario dell’Unità vedere, tra gli altri, Colombo 2011; Grassi Orsini 2011. 
resistenza contro lo sfruttamento piemontese. Sulla scia di queste interpretazioni revisioniste, quotidiani e siti internet hanno scatenato un'offensiva, che spesso è scaduta nel volgare, contro una storiografia ritenuta agiografica e filopiemontese [Baioni 2012, 15], provocando una serie di iniziative che hanno trovato modo di esprimersi in variazioni della toponomastica e nella rimozione di statue dedicate a personaggi al servizio della monarchia sabauda. Dopo essere stati a lungo dimenticati e guardati, spesso distrattamente, come meri elementi dell'arredamento urbano, i monumenti risorgimentali si sono così nuovamente trovati al centro del dibattito pubblico, pregni di una nuova forza simbolica. Presupponendo la volontà delle istituzioni di nascondere verità scomode riguardo la natura colonialista del processo di unità nazionale, alcune di queste ricostruzioni filoborboniche hanno concentrato le loro critiche sulle onorificenze rese al generale dell'esercito piemontese Enrico Cialdini (1811-1892). Cominciate con la decisione del Comune di Mestre, nel 2013, di cambiare nome a un piazzale cittadino a lui dedicato - progetto che ancora a oggi risulta irrealizzato -, le iniziative tese a cancellare il ricordo di Cialdini si sono rapidamente estese in città del Sud fino a richiedere la rimozione del suo busto presente alla Camera di commercio di Napoli.

Nato a Castelvetro, in provincia di Modena, ma cresciuto a Reggio Emilia, Cialdini attraversò tutta l'epopea risorgimentale. Cospiratore con Ciro Menotti nel 1831, si unì a Carlo Zucchi per poi espatriare e combattere in Portogallo e in Spagna. Rientrato in Italia nel 1848, prese parte alle guerre per l'indipendenza nazionale tra le fila dell'esercito piemontese. Nominato generale al rientro della Guerra di Crimea, nel 1860, comandò l'esercito piemontese nella battaglia di Castelfidardo - dove nel 1912 venne eretto un monumento in suo onore - e guidò l'assedio alla fortezza di Gaeta, che fece capitolare il 13 febbraio 1861. Nei mesi successivi, nel tentativo di domare le ultime sacche di resistenza borboniche e il brigantaggio, Cialdini si distinse per la durezza con la quale comandò una rappresaglia in seguito al massacro di 45 bersaglieri per mano di un gruppo di briganti e contadini armati a Casalduni (11 agosto 1861) in provincia di Benevento. Tre giorni più tardi, il 14 agosto, dietro comando di Cialdini, il colonnello Pier Eleonoro Negri (1818-1887) condusse due reparti contro i paesi di Casalduni, che fu però trovata quasi deserta, e la poco distante Pontelandolfo. Il bersagliere Carlo Margolfo ha lasciato una descrizione degli avvenimenti di cui fu partecipe.

$\mathrm{Al}$ mattino del mercoledì, giorno 14, riceviamo l'ordine superiore di entrare nel comune di Pontelandolfo, fucilare gli abitanti, meno i figli, le donne e gli infermi, ed incendiarlo. Difatti un po' prima di arrivare al paese incontrammo i briganti attaccandoli, ed in breve i briganti correvano davanti a noi. Entrammo nel paese: subito abbiamo incominciato a fucilare i preti ed uomini, quanti capitava, indi il soldato saccheggiava, ed infine abbiamo dato l'incendio al paese, abitato da circa 4.500 abitanti [Margolfo 1992, 53].

Già all'indomani della rappresaglia, i resoconti iniziarono a contraddirsi, rendendo difficile stimare il numero delle vittime. La più affidabile ricostruzione, 
effettuata tramite il registro dei defunti della parrocchia Santissimo Salvatore di Pontelandolfo, ha confermato con certezza la morte di 13 persone (11 uomini e due donne) [Panella 2000].

Nonostante la sua azione di repressione del brigantaggio del Mezzogiorno fosse risultata cruenta, Cialdini rimpianse amaramente l'ingrato compito affidatogli e, in guisa di riparazione, decise di devolvere alla città di Napoli il compenso percepito in quanto luogotenente del re per le province meridionali, vincolando una cifra consistente, corrispondente a 212,500 lire dell'epoca - divenute con gli interessi 1.300 lire nel 1893 - alla costruzione di una nuova sede della Borsa.

E l'amore della maggioranza del popolo al Cialdini è giustificato dalla gloria che lui spetta di aver dato nobilissimo esempio. [...] Egli ripartiva fra diverse istituzioni pubbliche e di beneficenza 451,500 lire, da lui risparmiate dalla somma assegnatagli per spese di rappresentanza. Tal somma divideva per la costruzione di una Borsa di commercio; per l'associazione filantropica intesa alla costruzione di case operaie; per sussidio alla Cassa di risparmio [...], per l'istituto delle fanciulle povere; per quello di beneficenza del Carminiello; per l'emigrazione veneta e romana; e per l'asilo infantile nel comune di S. Germano. [...] Cialdini aggiungeva un concetto altamente italiano, esprimendo, con sua lettera al presidente della Camera di commercio, il suo desiderio che nelle sale del nuovo edifizio della Borsa fosse eretto un busto a Cavour [Nisco 1892, 259-260].

Nonostante il beneventano Niccola Nisco giudicasse l'operato di Cialdini come «il quadro di un grande artista, nel suo insieme, in cui gli sprazzi di luce sono contrapposti da ombre sovente oscure» [Nisco 1892, 260], ad oggi, possiamo affermare che i fatti di Pontelandolfo e Casalduni figurino tra le pagine più nere del processo risorgimentale. In occasione del $150^{\circ}$ anniversario dell'Unità d'Italia, il sindaco di Reggio Emilia, Graziano Delrio, ha donato una replica del tricolore al Comune di Pontelandolfo, con l'intento di «ricordare verità storiche [...] non degne del processo unitario e restituire onore e giustizia a vittime innocenti» ${ }^{11}$. Tuttavia, il battage revisionistico è proseguito, trovando sponda nell'amministrazione comunale napoletana del sindaco Luigi De Magistris che, facendo seguito al ritiro della cittadinanza onoraria (19 aprile 2017), nel dicembre 2018, ha deliberato la rimozione del busto di Cialdini - da sostituire con una statua che ricordi Pontelandolfo - causando la protesta della Società napoletana di storia patria. Avanzare oltre rischierebbe di farci sconfinare nella cronaca ma, alla luce delle proteste che hanno portato alla rimozione dei memoriali e dei monumenti confe-

11 Comunicato stampa del Comune di Reggio Emilia, $150^{\circ}$ Unità d'Italia Il Primo Tricolore al Comune di Pontelandolfo (Benevento), teatro il 14 agosto 1861 di un eccidio dell'esercito sabaudo, 3 agosto 2011, https://www.comune.re.it/UfficioStampa/comunicatistampa.nsf/PESIdDoc/976B18 93BA0C95A7C12578E100504C0D/\$file/Sindaco\%20-\%20Pontelandolfo\%20-\%20consegna\%20 Primo\%20Tricolore\%20 3.8.pdf. 
derati negli Stati Uniti, la vicenda del busto di Cialdini impone una riflessione sul ruolo della statuaria risorgimentale nell'Italia di oggi. Ad essere messa in dubbio, non sembra essere l'unità della nazione, anche se non mancano rivendicazioni in tale direzione ${ }^{12}$ [Napoli 2019], ma l'interpretazione di un Risorgimento voluto anche dal basso, a favore di una visione colonialista del processo risorgimentale. Tuttavia, la motivazione profonda di questa «bonifica storica» sembra debba essere rintracciata in convinzioni politiche, o in problematiche attuali del territorio, proiettate retroattivamente nel passato, in modo da «estrapolare personaggi ed eventi dalla propria epoca, per renderli invece funzionali a problemi e questioni del presente» ${ }^{13}$. Questo processo interpretativo confonde un dato ben presente allo storico - le tracce e lo studio del passato aumentano la nostra comprensione del passato in quanto tale, e non presuppongono una condivisione dei valori che esso testimonia -, e tende a cancellare, oltre alle tracce fisiche del passato, la complessità degli eventi, banalizzandoli in un presente puntiforme. Le vestigia risorgimentali sono ritenute in toto antagoniste all'attuale identità territoriale, pensata come immodificabile nel tempo, a prescindere dalle motivazioni che condussero all'erezione di tali monumenti. Si ripropone così una distinzione tra "noi e loro", che la politica post-risorgimentale aveva cercato di ricomporre in un univoco spirito nazionale, destinata a mettere in discussione tutti i personaggi di quella travagliata epoca: la statua di Garibaldi può essere reinventata come simbolo di una conquista subita e la toponomastica cittadina alterata dai nostalgici degli Stati preunitari ${ }^{14}$. Quindi, se il monumento pubblico «diventa un segnale della lotta e dei punti di equilibrio fra le correnti vive del corpo sociale [...] sino quando, perduta ogni forza comunicativa, ridiventa pietra muta» [Isnenghi 1989, 337], il compito della statuaria risorgimentale è ancora lungi dall'essere compiuto. Come è servita in passato per costruire un'identità collettiva fondata sul ricorso alla memoria del processo d'indipendenza e degli sforzi da essa comportati, oggi, ad essa si deve associare un'interpretazione storica che, al contrario della memoria, non ha bisogno di cancellare o rinnegare le sue contraddizioni, permettendo di ricostruire il tortuoso, e non sempre limpido, cammino che ha portato alla nascita del nostro paese.

12 Napoli, striscione per la Festa dell'Unità: “Non riconosciamo l'Italia nazione”, in «La voce di Napoli», 17 marzo 2019, https://www.vocedinapoli.it/2019/03/17/napoli-striscione-per-la-festadellunita-non-riconosciamo-litalia-nazione.

13 Renata De Lorenzo, Togliere la statua del generale Cialdini stravolge la storia, in «La Repubblica», 23 febbraio 2019.

14 Francesco Palmieri, Vie, statue e sacrari: il Sud come l'America riscrive la storia nelle piazze, in «Agi», 17 agosto 2017, https://www.agi.it/cultura/usa sud italia monumenti charlottesville-2059110/news/2017-08-17/. 


\section{Bibliografia}

\section{Abeille 1879}

Antonio Abeille, Il IX gennaio 1878, ossia Il mondo civile ed in particolare l'Italia in morte di Vittorio Emanuele il grande, 2 voll., Napoli, Stabilimento tipografico Prete, 1879.

Agulhon 1978

Maurice Agulhon, La «statuomanie» et l'histoire, in «Ethnologie française», 2-3 (1978), pp. 145-172.

Angeli, Minardi 2014

Stefano Angeli, Alessandro Minardi, Quando l'Italia passò da Cesena: l'epopea risorgimentale di Eugenio Valzania, Cesena, Historica, 2014.

Baioni 2006

Massimo Baioni, Risorgimento in camicia nera. Studi, istituzioni, musei nell'Italia fascista, Roma, Carocci, 2006.

Baioni 2009

Massimo Baioni, Risorgimento conteso. Memorie e usi pubblici nell'Italia contemporanea, Reggio Emilia, Diabasis, 2009.

Baioni 2010

Massimo Baioni, Rituali in provincia. Commemorazioni e feste civili a Ravenna (1861-1975), Ravenna, Longo, 2010.

Baioni 2012

Massimo Baioni, Un mito per gli italiani. Il Risorgimento tra ricerca storica e discorso pubblico, in «Italian Culture», XXX, 2 (2012), pp. 7-20.

Balzani 1989

Roberto Balzani, Profilo di Eugenio Valzania, in «Studi romagnoli», XLIX (1989), pp. 291-300.

Balzani 1997

Roberto Balzani, Le tradizioni amministrative locali, in Storia d'Italia. Le regioni, 13, L'Emilia-Romagna, a cura di Roberto Finzi, Torino, Einaudi, 1997, pp. 599-646.

Balzani 2011

Roberto Balzani, Il debito di una regione col Risorgimento, in «... E finalmente potremo dirci italiani». Bologna e le estinte Legazioni tra cultura e politica nazionale, 1859-1911, a cura di Claudia Collina, Fiorenza Tarozzi, Bologna, Editrice Compositori, 2011, pp. 15-18.

Banti 2000

Alberto Mario Banti, La Nazione del Risorgimento. Parentela, santità e onore alle origini dell'Italia unita, Torino, Einaudi, 2000.

Banti, Ginsborg 2007

Storia d'Italia, Annali, 22, Il Risorgimento, a cura di Alberto Mario Banti, Paul Ginsborg, Torino, Einaudi, 2007.

Berselli 1980

Storia della Emilia-Romagna, a cura di Aldo Berselli, vol. III, Bologna, BUP, 1980.

Bizzoni 1883

Achille Bizzoni, Garibaldi narrato al popolo, Milano, Tipografia sociale E. Reggiani, 1883.

Brice 2004

Catherine Brice, La monarchie italienne et la construction de l'identité nationale, 1861-1911, Ph.D. thesis, Institut d’Études Politiques, 2004. 
Brice 2012

Catherine Brice, La monumentalità pubblica. Quale ricezione per il discorso politico nazionale nell'Italia di fine Ottocento?, in Parole in azione. Strategie comunicative e ricezione del discorso pubblico in Europa fra Otto e Novecento, a cura di Pietro Finelli, Gian Luca Fruci, Valeria Galimi, Firenze, Le Monnier, 2012, pp. 11-27.

Carducci 1938

Giosuè Carducci, Edizione nazionale delle opere di Giosuè Carducci, XXVIII, Bologna, Zanichelli, 1938.

Carrattieri 2012

Mirco Carrattieri, Monumento a Giuseppe Garibaldi, Guastalla, in Piraccini 2012, pp. 19-20.

Cattaneo 1860

Carlo Cattaneo, La circolare del ministro Farini sul riordinamento amministrativo, in «Il Politecnico», VIII (L) (1860), pp. 281-285.

Cavazza, Berardi 1980

Giulio Cavazza, Domenico Berardi, Il movimento repubblicano, in Berselli 1980, pp. 443-465.

Colombo 2011

Arturo Colombo, Storia e politica nei libri del $150^{\circ}$ dell'Unità italiana, in «Il Politico», 76, 3 (2011), pp. 335-371.

Corgnati, Mellini, Poli 1990

Il lauro e il bronzo. La scultura celebrativa in Italia, 1800-1900, Catalogo della mostra, a cura di Maurizio Corgnati, Gianlorenzo Mellini, Francesco Poli, Torino, s.n., 1990.

Della Peruta 1995

Franco Della Peruta, Il mito del Risorgimento e l'estrema sinistra dall'Unità al 1914, in Il mito del Risorgimento 1995, pp. 32-70.

d'Ideville 1878

Henry d'Ideville, Victor-Emmanuel II: sa vie, sa mort, souvenirs personnels, Paris, Palmé, 1878.

Documenti d'Archivio 2002

Documenti d'Archivio pubblicati in occasione del $140^{\circ}$ anniversario di Meldola Città, Meldola, Archivio storico comunale “Giacomo Zaccaria”, 2002.

Dumas 1882

Alexandre Dumas, Memorie di Giuseppe Garibaldi, seguite da un sunto storico fino alla morte del generale, Milano, Sonzogno, 1882.

Finale 2008

Finale, Garibaldi \& dintorni, Finale Emilia, s.n., 2008.

Galimi 2015

Valeria Galimi, Memorie di pietra: monumenti tra Siena e Grosseto fra Risorgimento e Grande Guerra, in Luoghi e simboli della memoria: le piazze della Toscana, a cura di Pier Luigi Ballini, Romano Paolo Coppini, Firenze, Regione Toscana, 2015, pp. 267-287.

Gavelli 2012

Mirtide Gavelli, Monumento a Giuseppe Garibaldi, Bologna, in Piraccini 2012, pp. 25-27.

Grassi Orsini 2011

Fabio Grassi Orsini, Introduzione, in «Ventunesimo Secolo», 26, 3 (2011), pp. 9-20. 


\section{Guerzoni 1882}

Giuseppe Guerzoni, Garibaldi, 2 voll., Firenze, Barbera, 1882.

Halbwachs 2001

Maurice Halbwachs, La memoria collettiva, Milano, Unicopli, 2001 (ed. or. 1950).

Il mito del Risorgimento 1995

Il mito del Risorgimento nell'Italia unita, Atti del convegno (Milano, 9-12 novembre 1993), in «Il Risorgimento», XLVII, 1-2 (1995).

Isnenghi 1989

Mario Isnenghi, Le guerre degli italiani: parole, immagini, ricordi, 1848-1945, Milano, Mondadori, 1989.

Isnenghi 2007

Mario Isnenghi, Garibaldi fu ferito. Storia e mito di un rivoluzionario disciplinato, Roma, Donzelli, 2007.

Isnenghi 1996-1997

I luoghi della memoria, a cura di Mario Isnenghi, 3 voll., Roma-Bari, Laterza, 19961997.

Levis Sullam 2010

Simon Levis Sullam, L'apostolo a brandelli. L'eredità di Mazzini tra Risorgimento e fascismo, Roma-Bari, Laterza, 2010.

Levra 1992

Umberto Levra, Fare gli italiani. Memoria e celebrazione del Risorgimento, Torino, Comitato di Torino dell'Istituto per la storia del Risorgimento italiano, 1992.

Lotti 1980

Luigi Lotti, La vita politica tra Ottocento e Novecento, in Berselli 1980, pp. 305-328.

Luzzatto 2011

Sergio Luzzatto, La mummia della Repubblica. Storia di Mazzini imbalsamato, Torino, Einaudi, 2011.

Körner 2009

Axel Körner, Politics of Culture in Liberal Italy. From Unification to Fascism, New York-London, Routledge, 2009.

Körner, Riall 2009

Axel Körner, Lucy Riall, Introduction: The New History of Risorgimento Nationalism, in «Nations and Nationalism», 15, 3 (2009), pp. 396-401.

Mantovani 2009

Luigi Davide Mantovani, Garibaldini ferraresi e la guerra del Veneto nel 1866, in, Garibaldi e il Polesine tra Alberto Mario, Jessie White e Giosuè Carducci, Atti del convegno di studi storici (Lendinara e Rovigo, 26-27 ottobre 2007), a cura di Zeffiro Ciuffoletti, Rovigo, Minelliana, 2009, pp. 189-218.

Margolfo 1992

Carlo Margolfo, Mi toccò in sorte il numero 15. Episodi della vita militare del bersagliere Margolfo Carlo, Delebio, Comune di Delebio, 1992.

Mario 1882

Jessie W. Mario, Vita di Giuseppe Garibaldi, 2 voll., Milano, Treves, 1882.

Mazzonis 2003

Filippo Mazzonis, La Monarchia e il Risorgimento, Bologna, il Mulino, 2003.

Miserocchi 2014

Lorenzo Miserocchi, Ravenna e i Ravennati nel Secolo XIX, Ravenna, Libreria Antiquaria Tonin, 2014 (ed. or. 1927). 
Morandi 2009

Matteo Morandi, Garibaldi, Virgilio e il violino. La costruzione dell'identità locale a Cremona e Mantova dall'Unità al primo Novecento, Milano, Franco Angeli, 2009.

Nasalli Rocca 1948

Emilio Nasalli Rocca, Atteggiamenti politici dei ducati emiliani e della città "primogenita” nel '48, Piacenza, Porta, 1948.

Nisco 1893

Niccola Nisco, Il generale Cialdini e i suoi tempi, Napoli, Morano Editore, 1893.

Palamenghi-Crispi 1912

Carteggi politici inediti di Francesco Crispi (1860-1890), a cura di Tommaso Palamenghi-Crispi, Roma, L’Universelle, 1912.

Panella 2000

Davide Fernando Panella, L'incendio di Pontelandolfo e Casalduni, 14 agosto 1861, in Brigante in terra nostra, San Giorgio del Sannio, Borrelli, 2000, pp. 77-114.

Pareti 2011

La Primogenita e i suoi Garibaldini, a cura di Stefano Pareti, Piacenza, L.I.R., 2011.

Parker 2011

Laura Parker, Identity, Memory and la Diarchia di Bronzo. Commemorating Vittorio Emanuele II and Giuseppe Garibaldi in Post-Risorgimento Venice, in «Incontri», XXVI, 2 (2011), pp. 25-36.

Piraccini 2012

Monumenti Tricolori. Sculture celebrative e lapidi commemorative del Risorgimento in Emilia e in Romagna, a cura di Orlando Piraccini, Bologna, Editrice Compositori, 2012.

Porciani 1993

Ilaria Porciani, Stato, statue, simboli: i monumenti nazionali a Garibaldi e a Minghetti del 1895, in «Storia, Amministrazione, Costituzione», I (1993), pp. 211-242.

Riall 2007

Lucy Riall, Garibaldi. Invention of a Hero, New Haven-London, Yale University Press, 2007.

Ridolfi 1989

Maurizio Ridolfi, Il partito della Repubblica. I repubblicani in Romagna e le origini del Pri nell'Italia liberale (1872-1895), Milano, Franco Angeli, 1989.

Romanelli 1989

Raffaele Romanelli, La nazione e il campanile. Il dibattito intorno alle dimensioni dello scambio politico nell'Italia liberale, in Gli spazi del potere. Aree, regioni, Stati: le coordinate territoriali della storia contemporanea, a cura di Franco Andreucci, Alessandra Pescarolo, Firenze, La Casa Usher, 1989, pp. 184-191.

Rossi 2010

Lauro Rossi, Garibaldi: due secoli di interpretazioni, Roma, Gangemi, 2010.

Rotelli 1980

Ettore Rotelli, Gli ordinamenti locali preunitari, in Berselli 1980, pp. 231-256.

Spadolini 1995

Giovanni Spadolini, Mito ed eredità del Risorgimento, in Il mito del Risorgimento 1995, pp. 5-12.

Struttura e dinamica delle unità amministrative territoriali italiane 2018

Struttura e dinamica delle unità amministrative territoriali italiane: dall'unificazione del Regno al 2017, Roma, Istat, 2018. 


\section{Tobia 1991}

Bruno Tobia, Una patria per gli italiani. Spazi, itinerari, monumenti nell'Italia unita, 1870-1900, Roma-Bari, Laterza, 1991.

Tobia 1995

Bruno Tobia, Una forma di pedagogia nazionale tra cultura e politica: i luoghi della memoria e della rimembranza, in Il mito del Risorgimento 1995, pp. 174-214.

Varni 1995

Angelo Varni, Il secondo Risorgimento, in Il mito del Risorgimento 1995, pp. 535-543.

Varni 1997

Angelo Varni, I caratteri originali della tradizione democratica, in Storia d'Italia. Le regioni, 13, L'Emilia-Romagna, a cura di Roberto Finzi, Torino, Einaudi, 1997, pp. 553-575.

Varni 2011

Angelo Varni, Ripensando all'eroe: Giuseppe Garibaldi, in Dialogo con le personalità del Risorgimento: convegni del $150^{\circ}$ dell'Unità d'Italia (Bologna, 25 marzo 2011), Bologna, BUP, 2011, pp. 33-47.

Zanni Rosiello 1965

Isabella Zanni Rosiello, L'unificazione politica e amministrativa nelle "Provincie dell’Emilia” (1859-1860), Milano, Giuffrè, 1965.

Zanni Rosiello 2000

Isabella Zanni Rosiello, I moderati emiliani e i problemi legislativi e amministrativi delle "Provincie dell'Emilia" dopo l'annessione, in L'archivista sul confine. Scritti in onore di Isabella Zanni Rosiello, a cura di Carmela Binchi, Tiziana Di Zio, Roma, Ministero per i beni e le attività culturali, Ufficio centrale per i beni archivistici, 2000, pp. 27-65. 